\title{
The small reconnaissance of atmospheres mission platform concept, part 1: motivations and outline for a swarm of scientific microprobes to the clouds of Jupiter in 2030
}

\author{
John E. Moores* \\ Centre for Research in the Earth and Space Sciences (CRESS), \\ York University, \\ 4700 Keele Street, Toronto, ON M3J 1P3, Canada \\ Email: jmoores@yorku.ca \\ *Corresponding author \\ Kieran A. Carroll \\ University of Toronto Institute for Aerospace Studies, \\ University of Toronto, \\ Canada \\ Email: Kieran.carroll@gedex.com
}

Isaac DeSouza, Kartheephan Sathiyanathan, Barry Stoute, Jinjun Shan, Regina S. Lee and

Ben Quine

\author{
CRESS, \\ York University, \\ Canada \\ Email: desouzai@yorku.ca \\ Email: kartheep@yorku.ca \\ Email: bstoute@yorku.ca \\ Email: jjshan@yorku.ca \\ Email: reginal@yorku.ca \\ Email: bquine@yorku.ca
}

\begin{abstract}
A mission concept is presented for several small atmospheric entry vehicles at Jupiter. By relaxing the requirement for substantial penetration into the Jovian atmosphere, the size of the atmospheric entry probes shrinks dramatically. Such atmospheric entry probes would experience much less heating than previous concepts of much larger $(\sim 300 \mathrm{~kg})$ spacecraft presented as minimum concepts and no parachutes are necessary. This reduces complexity while still permitting over 15 minutes of useable science under free-fall from above the 0.41 bar level to near the 10 bar level of the Jovian Atmosphere during which up to 20 Mbits of data could be returned per probe. By dividing the payload, the risk to the mission is substantially mitigated and ground truth may be obtained from a large part of the entire planetary atmosphere using a single launch.
\end{abstract}


Keywords: mission concept; spacecraft design; planetary science; atmospheric science; Jupiter; microprobe.

Reference to this paper should be made as follows: Moores, J.E., Carroll, K.A., DeSouza, I., Sathiyanathan, K., Stoute, B., Shan, J., Lee, R.S. and Quine, B. (2014) 'The small reconnaissance of atmospheres mission platform concept, part 1: motivations and outline for a swarm of scientific microprobes to the clouds of Jupiter in 2030', Int. J. Space Science and Engineering, Vol. 2, No. 4, pp.327-344.

Biographical notes: John E. Moores is an Assistant Professor of Space Engineering at York University.

Kieran A. Carroll is an Adjunct Research Professor at the University of Toronto.

Isaac DeSouza is an undergraduate in the Space Engineering program at York University.

Kartheephan Sathiyanathan is a doctoral candidate in the Earth and Space Science program at York University.

Barry Stoute is a recent graduate of the Earth and Space Science program at York University.

Jinjun Shan is an Associate Professor of Space Engineering at York University.

Regina S. Lee is the Chair of the Department of Earth and Space Science and Engineering at York University.

Ben Quine is a Professor of Space Engineering and Planetary Physics at York University.

\section{Introduction}

\subsection{Microsats for planetary science}

Over the last 20 years, advances in miniaturisation and electronics design have made practical a completely new class of small space vehicle in near earth orbits. Such micro, nano and cube satellites, some with masses of a $\mathrm{kg}$ or less, have lowered the cost of access to space and have enabled many groups who were previously economically restrained from participating in larger missions to design their own satellites end to end in order to do small-scale science and test engineering advances on-orbit.

However, this revolution in near-earth space has not, thus far, extended to missions beyond GEO. There are several compelling reasons for this. First, the small size of microsats limits the amount of power they may acquire from solar arrays. This problem becomes especially acute at large distances from the sun. Secondly, such satellites are limited by their low mass in the variety of payloads they may carry. Most critically, the large distances from the Earth required for interplanetary missions require large high gain antennas (HGAs), powerful transmitters, and large ground stations in order to be practical. Furthermore, the existing infrastructure is not conductive to interplanetary 
microsats (Lang et al., 2013). While the orbital mechanics are favourable - there would be distinct advantages in accelerating a small payload to interplanetary transfer velocities - such small-scale upper stages do not yet exist. As well there are regulatory hurdles. For instance, while nothing restricts the minimum size of a radioistope thermoelectric generator (RTG) unit, international regulations currently restrict the production of Pu238-powered devices that puts these out of reach for all but US and Russian government space agencies. This said, there is a desire, at least within the US, to develop smaller RTGs for use on small deep space vehicles (Bairstow and Cataldo, 2013). Such devices would enable NASA-led small planetary missions.

Despite this environment, there are certain niche applications in planetary exploration that could benefit from the microsat concept (Worden, 2012). Where microsats must work independently communication over interplanetary distances are the largest hurdle. Where time on large ground-based radio telescope in the $30-\mathrm{m}$ to $70-\mathrm{m}$ class may be obtained, missions to the moon (Garrick-Bethell et al., 2013) or to near-earth asteroids (e.g., Riedel et al., 2013; Bairstow and Cataldo, 2013; Carroll et al., 2012) or to investigate the near interplanetary environment (Lloyd, 2013) become practical. As well, several missions have been proposed which are intended to make use of more substantial assets already deployed. These include several missions at Mars, including magnetometric mapping orbiters (Spencer and Zee, 2012), missions to the moons Phobos and Deimos (Castillo-Rogez et al., 2013) swarms of geophysical and meteorological stations in orbit (Komarek et al., 2013) or on the surface, and fleets of small rovers. Lastly, the use of small cubesat sized ancillary vehicles to augment the work of larger planetary vehicles (Poncy et al., 2013; Halatek, 2013) presents an exciting new opportunity to maximise the science return of the existing planetary exploration paradigm.

The key to all of these proposals is the clever utilisation of pre-existing assets. At Mars, this consists of the large number of in-situ orbiters with relay capability (Mars Express, Mars Reconaissance Orbiter, Mars Odyssey) that reduces the distance over which the microsat or ground station must communicate to a few hundred to a few thousand kilometres. The question to be addressed in this paper is whether the anticipated presence of ESA's JUICE orbiter in the Jovian system, starting in 2030, would likewise enable tandem microsat missions and tandem science to be performed at Jupiter.

\subsection{Opportunities for microsats at Jupiter?}

In particular, the feasibility of several atmospheric probes, each of which could be potentially contributed by different organisations, will be considered. Given the extremely high entry velocities at Jupiter, the aerothermodynamics of high surface area/low mass probes heavily favour such small craft over vehicles in the Galileo Entry Probe class and larger which require in excess of $50 \%$ of their mass to be devoted to shielding in order to survive entry. Furthermore, once in the atmosphere, the low terminal velocity of such small vehicles allows designs to avoid the use of parachutes, substantially decreasing the complexity of these vehicles.

There is a great deal of valuable science yet to be accomplished at Jupiter. The 2011 Planetary Decadal Survey (NRC, 2011) which synthesised the science priorities of the Planetary Science Community cited Jupiter's atmosphere as an object of study under two of its three top level themes: 'building new worlds' and 'workings of the solar system'. Furthermore, the survey identified its third priority amongst medium class (New 
Frontiers) missions as 'Jupiter Polar Orbiter with Probes' (NRC, 2011). While the Juno mission (Matousek et al., 2007) will cover the first half of this priority investigation, the science of the orphaned probe investigations could be achieved through a separate mission. Furthermore, the report notes that even at shallow depths, the atmospheres of Giant planets are subject to dramatic changes that were unknown following the Galileo Probe mission (NRC, 2011). Such change brings into question whether the atmosphere may be considered well-mixed and, therefore, how representative the single data point of the Galileo entry probe is of this largest reservoir of planetary building material in the solar system.

There are also engineering and exploration reasons for revisiting Jupiter. For instance, the Galileo Entry Probe did not carry a camera or an UV/Visible/NIR spectrometer. Such instruments could revolutionise our understanding of the nature of the clouds and of dynamic atmospheric processes too small to be observed from orbit or hidden beneath the upper cloud decks. A photograph from within the atmosphere of a giant planet is also one of the few remaining relatively easily obtainable 'firsts' in our exploration of the solar system and addresses the lack of adequate spatial resolution at Jupiter for understanding atmospheric phenomenon in currently approved spacecraft (NRC, 2011). This resolution is limited to $15 \mathrm{~km}$ per pixel and will be achieved by JunoCam onboard the Juno spacecraft (Matsousek, 2007). Furthermore, by employing multiple entry probes, several different sites may be sampled. While the spread between probes cannot be too large in order for the communications scheme to be effective, this network approach may avoid one of the pitfalls of the Galileo Mission whose one point of data is thought to correspond to an atypical part of the atmosphere (Orton et al., 1996). Finally, by dividing up the mission into several separate individual spacecraft, the risk inherent in an atmospheric entry may be somewhat mitigated with each customer able to choose their own level of comfort with respect to the technology readiness level (TRL) of their own hardware. This is a common solution employed for cubesats today in which many independent spacecraft are launched by a single vehicle (Lang et al., 2013).

As such, this paper will present a potential mission concept to advance Jovian science using a small spacecraft taking advantage of the presence of JUICE along with the necessary orbital mechanics and the key parameters of critical subsystems. Finally, we will examine a potential design for the entry probes along with several prospective payloads. This mission platform is designated as SMARA for SMAll Reconnaissance of Atmospheres. The name comes from the samara fruit of the maple tree, whose simple, small and light design allows it to catch the wind and fall gently through the atmosphere without the need of a parachute.

\section{Motivation: atmospheric science at Jupiter}

\subsection{Overview of science at Jupiter}

Motivation to study of the Atmosphere of Jupiter comprises three different thrusts. The first relates to composition and solar system history: over two thirds of all solar system mass that is not part of the sun is incorporated into Jupiter. As such, Jupiter provides an invaluable resource to understand the early solar nebula and hence the initial conditions from which all the planets formed. In particular, the results of the Galileo Probe found 
surprising abundances of noble gasses that are significant for models of the early solar system and constrain the processes that form the planets as well as the internal differentiation of Jupiter itself (NRC, 2011). Furthermore, Jupiter is subject to continuous bombardment by small bodies that provide an exogenous input to the modern-day stratosphere. De-convolving this exogenous contribution would allow better estimations of the solar nebula contribution to the composition of Jupiter. Such investigations would also provide benefits to assessing the population of small impactors now and in the past. Thus, as highlighted by the Planetary Decadal Survey "a better inventory of Jovian stratospheric composition along with improved atmospheric models and numerical models of asteroid and comet orbits would constrain impact history", in fact, it is possible that the shallow Jovian atmosphere may even "be utilized as a record of impact history" (NRC, 2011).

Secondly, Jupiter provides the most accessible example of a deep atmosphere in our own solar system. As such, Jupiter's atmosphere represents a laboratory under which we can study flow dynamics, cloud microphysics and radiative transfer under conditions significantly different from those we encounter on earth and the other terrestrial planets. In fact, the decadal survey (NRC, 2011) points out that "the stratospheric oscillations that have been discovered on Earth, Mars, Jupiter and Saturn provide a rare stage for conducting comparative planetological investigation between terrestrial and giant planets". Understanding how the atmosphere of Jupiter works can therefore lead to improved understanding of the physics underlying our own atmosphere and can help to improve models predicting short term effects, such as weather and the movement and intensification of storms, and long-term effects such as climate change.

Finally, as the most accessible representative of a Giant Planet, Jupiter allows us to study more generally the processes that affect this class of bodies. Such planets are now known to exist beyond our own solar system and such extra-solar giant planets around other stars now count nearly one thousand known members, a list that is growing rapidly. The Decadal Survey highlighted this 'local laboratory' capability advising, in particular, studies of "heat flow and radiation balance" and "chemistry of giant planet atmospheres" (NRC, 2011).

\subsection{In-situ science and the Galileo entry probe}

The Science performed by the Galileo Entry Probe is summarised by Young et al. (1996) and the mission itself as well as the onboard experiments is described by Hunten et al. (1986). In short, the Galileo Entry probe made the first, and so far only, entry into the atmosphere of a gas giant planet. Despite entering a non-representative hot spot (Orton et al., 1996) it made valuable in-situ measurements. The frequency of lightning within several earth radii of the probe was measured (Lanzerotti et al., 1996). The temperature and pressure of the atmosphere from entry past heat shield separation at 0.41 bars to failure near 24 bars was measured (Seiff et al., 1996) along with the bulk composition of the atmosphere (Niemann et al., 1996), the nature and density of aerosols (Ragent et al., 1996, 1998), the spectrum of radiative transfer (Sromovsky et al., 1996), the speed of the winds at both high (Beebe et al., 1996) and deep levels (Atkinson et al., 1996) and the Helium Mass fraction was measured (Von Zahn and Hunten, 1996). Just prior to atmospheric entry, the inner radiation belts of Jupiter were probed (Fischer et al., 1996). 
There were several surprises. No water cloud layer was observed and aerosols appeared to be present throughout the descent (Ragent et al., 1996, 1998; Sromovsky et al., 1996). The Helium and Xenon abundances were much higher than models suggested (Von Zahn and Hunten, 1996; Niemann et al., 1996), the Oxygen and Neon abundances were both much lower than anticipated (Niemann et al., 1996) and the thermosphere was much hotter and denser than previously anticipated (Seiff et al., 1996). While the atmosphere was dynamically stable, except for gusts at the 2.2 and 3.3 bar level, strong zonal winds persisted to deep levels (Atkinson et al., 1996) indicating that Jovian weather is not a thin skin phenomenon. This suggests an interior power source significantly contributed to the observed movement of the atmosphere. However, given the high Helium abundance, this is unlikely to be gravitational potential energy from a separation of Helium from Hydrogen, as is suspected from analysis of Saturn (Fortney and Hubbard, 2003). Measurements of the isotopes of Helium and Hydrogen have allowed refinement of the character and composition of the solar nebula (Niemann et al., 1996).

\subsection{What remains to be done in-situ}

However, much remains to be done in-situ at Jupiter. Unfortunately, the nonrepresentative nature of the Galileo Entry Point (Orton et al., 1996) demands a follow-up mission to sample portions of the planet with a more representative atmosphere to put its results into context. This is especially true of the cloud structure. Water clouds have yet to be directly observed (Young et al., 1996) and the chemical aerosol or gas responsible for the coloration of the bands is poorly understood. As well, there is a need for a better oxygen abundance (Balint, 2005) to within $10 \%$ in order to properly constrain formation and evolution models of the planet. Furthermore, sampling different zonal bands near the equator would allow better models for the atmosphere to be created. Lastly, the strong winds at deep levels suggest that retrievals from even deeper zones may yield data that further constrains the internal motion and structure of Jupiter. This data would greatly enhance the gravity measurements to be obtained by the Juno mission in 2018 and motivates some of the deep probe proposals (e.g., Balint, 2005).

Unfortunately, in order to measure the oxygen abundance, present mainly as water, in-situ it is necessary to descend to a well-mixed layer below the clouds (Atreya and Wong, 2005). For Jupiter, this may be encountered as early as 9 bars, but more likely at 50-100 bars. However, nearly every other species will be well mixed in the upper 10 bars and will be accessible to a shallow-entry probe. It is for this reason that Atreya et al (2006) recommend multiple shallow probes to Jupiter and Saturn coupled with microwave radiometry from orbit or via flyby to obtain the water and hence the oxygen abundance. Juno will perform much of this microwave radiometry. By drastically increasing the number of locations sounded by shallow probes, a comparison dataset to this water abundance would be created that would yield a complete and global picture of the chemistry and dynamics of the Jovian atmosphere. 
Figure 1 Cartoon showing SMARA mission profile (see online version for colours)

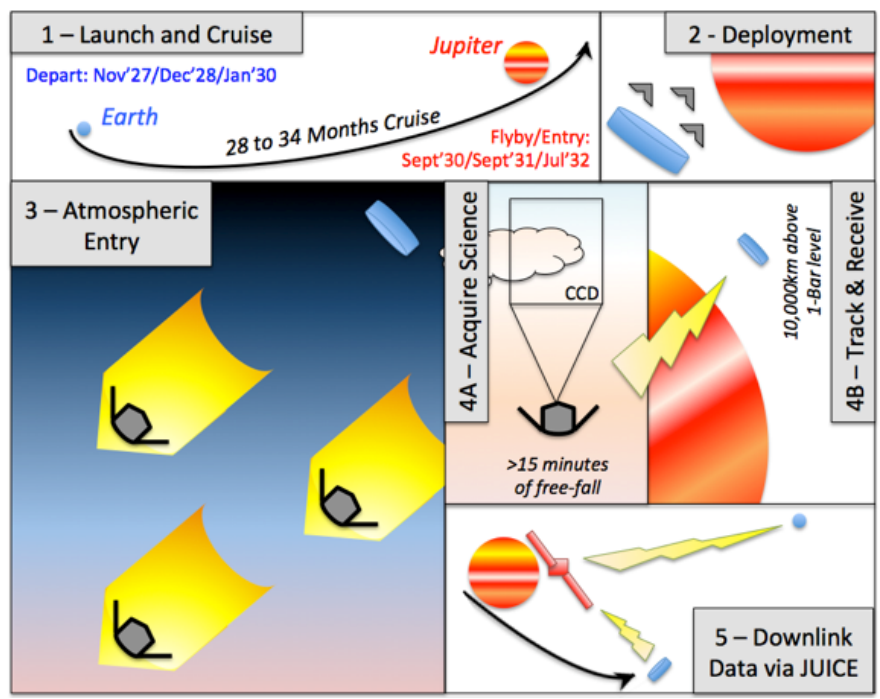

Notes: Beginning with Launch and cruise (1), the atmospheric entry probes (grey angles) are deployed from the carrier spacecraft (blue cylinder) prior to the Jovian Encounter (2). The probes orient themselves for atmospheric entry and rapidly slow to terminal velocity (3). During the 15 minutes of fall, the probes collect science and communicate back to the carrier spacecraft (4). Finally, following the encounter as the carrier continues on a hyperbolic path that will take it out of the Jovian system, it commences a rapid downlink of data to the JUICE spacecraft (shown in Red) which relays this data on to Earth (5).

\section{Overall mission profile}

\subsection{Concept}

The mission profile concept is illustrated in Figure 1 and is described below. Following launch in November of 2027, a carrier spacecraft with several small atmospheric probes enters cruise along a direct Hohmann trajectory to Jupiter. The carrier spacecraft is equipped with a small (2m-class) HGA that provides periodic health-check communications between the carrier and the Earth through the deep space network (DSN) during cruise at low data rates over the $\mathrm{S}$ or $\mathrm{X}$ radio bands. The craft also is equipped with a small solar array to provide power for these infrequent low-power transmissions and to power a small ion engine. During cruise the probes are dormant except for periodic short duration health-check wakeups commanded by the carrier electronics.

Prior to in-system arrival in September of 2030, the probes are ejected from the carrier as a swarm with enough impulse that they intersect the upper atmosphere of Jupiter. Once they hit the top of the atmosphere, these entry probes decelerate using ablative heat shields until they achieve terminal velocity in the vertical direction and slow to mean atmospheric velocities in the horizontal direction.

At this point the probes begin acquiring scientific measurements and will drop through the atmosphere, reaching the 10 bar level approximately 15 minutes after peak 
deceleration. While the fall of the probes is not actively controlled with a parachute, their mass distribution is such that they achieve a stable configuration as they fall through the atmosphere. Each probe may contain a different instrument payload such that the sum of their measurements is greater than what could be achievable with a single entry vehicle. Eventually, the probes fail as they encounter high pressures and temperatures.

Meanwhile, the carrier spacecraft continues on a hyperbolic trajectory that approaches within 10,000 km of the 1 bar level of Jupiter. During this mission phase, the carrier and its attached HGA slew to track the probes descending through the atmosphere. Once the probes have failed or the carrier has passed over the local horizon with respect to the probes, the carrier slews to track the JUICE spacecraft and begins downlinking the data received from the probes before departing the Jovian system. JUICE then downlinks this data directly to Earth through the DSN.

\subsection{Orbital mechanics and trajectories}

\subsubsection{Cruise}

The baseline Hohmann orbit and the corresponding porkchop plot showing launch opportunities to Jupiter is provided in Figure 2. Other, less fuel intensive trajectories may be possible using combinations of Earth and Venus gravity assists, as other spacecraft to the outer planets have utilised. In order to arrive at Jupiter during the JUICE primary mission, there are three separate launch windows. These occur in November of 2027, December of 2028 and January of 2030. For each of these opportunities, the minimum excess $\Delta v$ required to achieve a Jovian transfer orbit from an orbit with a $C_{3}$ of 0 is 6.30 , 6.25 and $6.20 \mathrm{~km} / \mathrm{s}$, respectively. This energy must be provided by the upper stage of the launch vehicle or by a separate apogee engine mated to the carrier within the payload fairing.

Figure 2 Porkchop plot for the 2028 departure window showing the relationship between time of flight and launch date (see online version for colours)

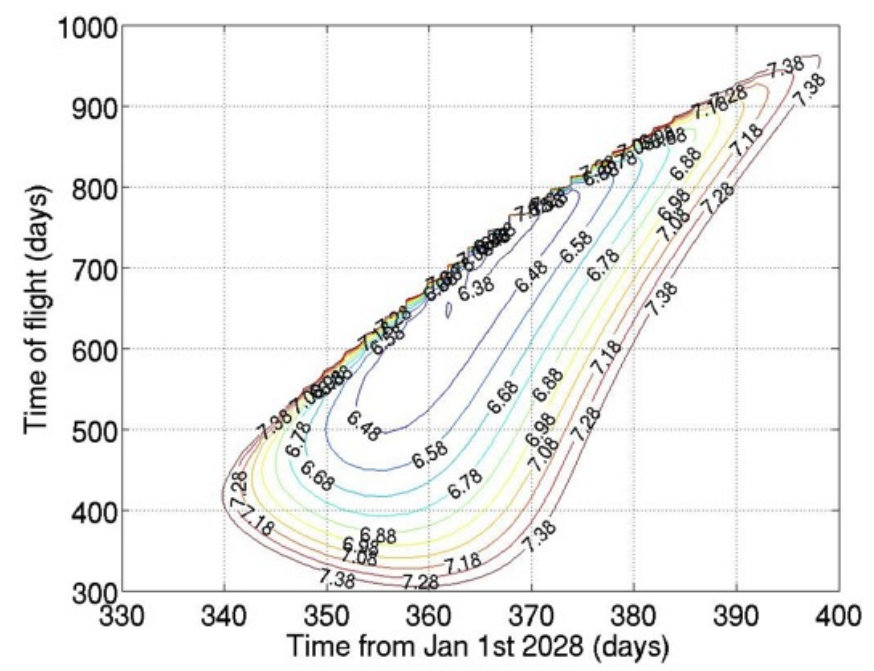

Notes: The 2027 and 2030 departures produce similar plots. The minimum value of the excess departure velocity is $6.25 \mathrm{~km} / \mathrm{s}$. 
Note that this analysis does not include $\Delta v$ budget for perturbations nor for a likely broken-plane manoeuvre. Generalising from past examples (e.g., Mitri, 2002) this additional requirement for $\Delta v$ en-route is likely to be in the range of $300 \mathrm{~m} / \mathrm{s}$, including margin. Part of this could be used to fine-tune the geographic entry point of the probe. This is an area in which JUICE provides a substantial benefit - by providing imagery and spectroscopy of the planet prior to arrival, the carrier spacecraft could be steered away from any hot spots and thereby samples a more representative upper atmosphere (Young et al., 1996; Orton et al., 1996).

\subsubsection{Encounter trajectories}

This cruise trajectory will result in velocities of 65.1 to $64.8 \mathrm{~km} / \mathrm{s}$ at perijove for the carrier spacecraft, depending on which launch window is chosen. Due to the rapid equatorial rotation of Jupiter of $12.6 \pm 0.5 \mathrm{~km} / \mathrm{s}$, the entry probes will experience a velocity relative to the atmosphere of $52.4 \pm 0.5 \mathrm{~km} / \mathrm{s}$. The uncertainty here is due to the difference in equatorial zonal wind speeds and will depend on the latitude of entry. While different cruise trajectories can reduce this velocity marginally, even the limiting case of a parabolic entry into the Jovian system (i.e., $C_{3}=0$ with respect to Jupiter) would have an encounter speed of $59.5 \mathrm{~km} / \mathrm{s}$ and hence the relative entry velocity of the probes would be no less than $46.9 \pm 0.5 \mathrm{~km} / \mathrm{s}$.

The descent of the entry probes, as a function of time, is provided in Figure 3. In producing this figure, the following assumptions have been made. First, is assumed that the probes reach terminal velocity by at the latest the 0.4 bar pressure level and remain at terminal velocity throughout their descent, as was the case for the Galileo Entry Probe. It is likely, based upon the much lower ratio of mass-to-surface area that terminal velocity will be achieved sooner at lower pressure and higher altitude, however a full aerothermodynamics analysis is beyond the scope of this paper (please see Moores et al. (2014) for a simplified analysis). Further, it is assumed that each probe has a mass, $m$, of $12.5 \mathrm{~kg}$, that the diameter of the heat shields are $60 \mathrm{~cm}$ and that the heat shield is near conical in shape, providing a coefficient of drag, $C_{D}$, of approximately 0.5 . Lastly, the profile of temperature, $T$, versus height, $z$, in the Jovian atmosphere retrieved from the Galileo Entry Probe is also assumed (Seiff et al., 1996) and may be approximated above the 24 bar pressure level, i.e., above the failure pressure of the Galileo Entry Probe, as:

$$
T \cong-1.78 \times 10^{-3} z+168 . K
$$

where $T$ is in $K$ and $\mathrm{z}$ is in $\mathrm{m}$ with respect to the 1 bar pressure level. This permits calculation of the density profile of the atmosphere, further assuming that the atmosphere is composed entirely of hydrogen with a specific gas constant of $4,157 \mathrm{~J} \mathrm{~kg}^{-1} \mathrm{~K}^{-1}$ and assuming the ideal gas law over the fall region of interest. The terminal velocity at each level may be calculated by balancing the drag and gravitational forces on the spacecraft:

$$
\frac{d z(\rho)}{d t}=\sqrt{\frac{2 m g}{\rho A C_{D}}}
$$

where the 1 bar gravitational acceleration, $g$, of $23.12 \mathrm{~m} \mathrm{~s}^{-2}$ is assumed constant over the $\sim 100 \mathrm{~km}$ fall distance to the 10 bar level. The 10 bar pressure level was selected since it is (1) below the level of atmospheric aerosols and (2) provides a less stringent 
requirement on the design of the probes themselves compared to the Galileo entry probe, allowing these smaller probes to be produced and validated in a more economic fashion.

Applying the assumptions above to this equation yields the terminal velocity of the spacecraft as a function of altitude above the 10 bar pressure level as a function of altitude and time, shown graphically in Figure 3. Integrating this curve suggests a fall time of 1080 seconds between the 0.4 and 10 bar pressure levels. Note that the rate of descent of the spacecraft slows considerably as it descends, ranging from $210 \mathrm{~m} / \mathrm{s}$ near the condensation level of ammonia clouds at the 0.5 bar level to $90 \mathrm{~m} / \mathrm{s}$ near the condensation level of water ice clouds near the 6 bar level and finally $75 \mathrm{~m} / \mathrm{s}$ at the $10 \mathrm{bar}$ level. Thus, should the probes survive to lower altitudes, the amount of time spent in the atmosphere will increase dramatically.

Figure 3 Probe altitude, temperature and pressure as a function of time after attaining the 0.41 bar pressure level

\section{Atmospheric Entry Probe fall profile}

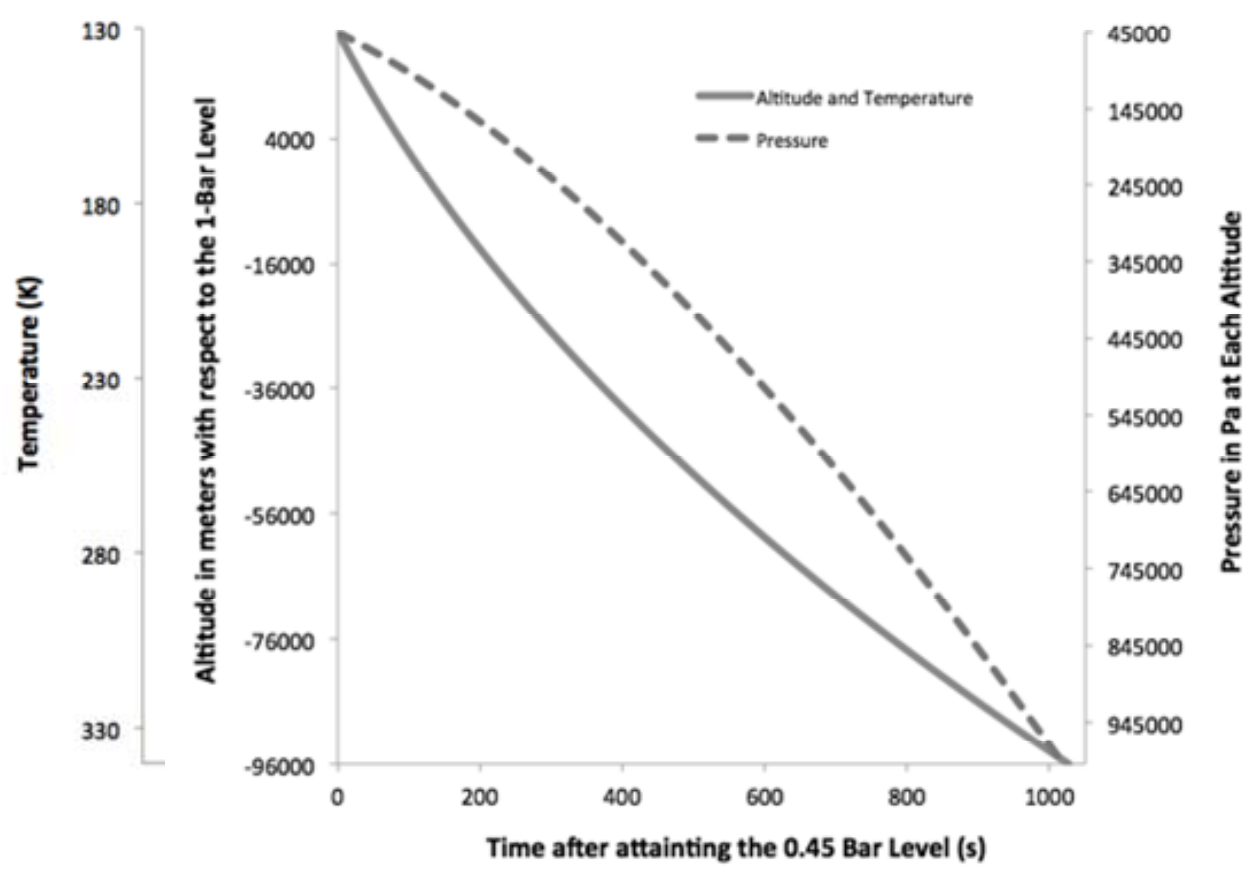

Notes: The probes reach ultimate values of $10 \mathrm{bar}$, and $340 \mathrm{~K}\left(67^{\circ} \mathrm{C}\right)$ after 1150 seconds of free-fall at terminal velocity.

Once the probes have slowed to atmospheric velocity in the horizontal direction and have begun their descent, the carrier spacecraft will have a relative velocity, with respect to the probes, of $52.4 \pm 0.5 \mathrm{~km} / \mathrm{s}$ and will continue on its hyperbolic path. This means that if the carrier spacecraft is directly overhead of the probes at $10,000 \mathrm{~km}$ above the 1 bar level halfway through their fall at $\sim 540$ seconds the most rapid data rate will be available as the probes enter the water cloud layer, the deepest of the condensable species. This would mean that the elevation of the carrier spacecraft, with respect to the probes, would be $21^{\circ}$ 
when the probes are at the 0.41 and 10 bar levels and the transmit distance would be $28,000 \mathrm{~km}$. Since passage through higher clouds would occur before the half-time of the fall, it might be preferable to bias the highest elevation angles to earlier during the passage, at the cost of loss of signal due to low elevation of the carrier spacecraft with respect to the probes occurring prior to probe failure.

The transmit distance above is high for a small transmitter and may not be realistic. This begs the question of how close the carrier spacecraft may safely approach the 1 bar level of Jupiter's atmosphere. For spacecraft in LEO, standard parking orbits begin at $185 \mathrm{~km}$ where the pressure is $1 \times 10^{-9} \mathrm{bar}$. Using the data from the Galileo Entry probe (Seiff et al., 1996), for Jupiter, this level is at approximately $1,000 \mathrm{~km}$ above the $1 \mathrm{bar}$ level. Therefore, approaching closer than this level is likely to be unsafe. Note that this is substantially closer than the Juno Orbiter that will pass between 4,000 km and 5,000 km above the 1 bar level at its closest point of approach (Matousek, 2007). However, given the single flyby trajectory of the carrier spacecraft, lower orbits should be achievable without resulting in excessive orbit degradation during the single pass. A note of caution is required, however - the Galileo Entry Probe's reported values at the $1000 \mathrm{~km}$ level were two orders of magnitude larger than anticipated (Seiff et al., 1996) and very little is known regarding temporal variations in pressure at this level of the atmosphere.

\subsubsection{Downlink trajectory}

Following the passage of the probes through the atmosphere, the carrier spacecraft will rotate and relay its data across to the JUICE spacecraft. It is likely that JUICE would be out beyond the orbit of Ganymede, at least 1,000,000 km from the position of the probes and the carrier spacecraft, in order to minimise its radiation exposure. Thus the ideal scenario would have JUICE's position on its orbit advanced beyond that of the carrier spacecraft. The carrier would then catch up to and overtake JUICE on its way out of the Jovian system. This would allow the range between the two spacecraft to decrease to a minimum, aiding data throughput.

It is likely that JUICE would also wish to observe any ionisation in the atmosphere of Jupiter as the result of the passage of the probes. This adds an additional constraint to the positioning of the spacecraft since it would require the probes to enter the atmosphere after JUICE has risen above the local horizon by at least $30^{\circ}$.

\subsection{Communications scheme}

More than any other subsystem, communications requirements - in particular during the encounter phase - drive not only the design of this individual subsystem on each spacecraft, but also drive the overall mission profile. Furthermore, the communications scheme describes an interaction between different payload elements along with existing assets in the Jovian system (e.g., JUICE or Europa Clipper) and on the ground. Therefore, an introduction to these linkages is provided here, beginning with describing the Encounter phase in Section 3.3.1 and demonstrating how hardware that satisfies this phase of the mission will automatically satisfy the Cruise and Downlink phases. The specific design of hardware on each individual spacecraft is left to the following issue (Moores et al., 2014). 


\subsubsection{Encounter}

During the encounter, the individual atmospheric entry probes need to be able to relay their data for eventual transmission to Earth. No communications are anticipated during the initial entry due to disruption caused by atmospheric ionisation. However, once the friction on the heat shields of each probe subsides below the ionisation limit, communications will once again become possible. Since each probe is subject to unpredictable atmospheric forces as the result of a potentially gusty atmosphere, highgain communications are not possible directly from each probe.

Instead, a hemispherical ( $2 \pi$ str) low gain antenna is selected. This design assumes that the probe configuration will be sufficiently bottom heavy that flipping or tumbling behaviour would be unlikely. While the eventual dynamics may permit a more focused transmission angle if the probes have increased stability, this transmitter design represents a reasonable worst case. An example of a microsat that uses this scheme is the MOST spacecraft which employs a $180 \mathrm{~g}, 6 \mathrm{~W}, 2.4 \mathrm{GHz}$ (S-band) hemispherical LGA with a transmit power of $0.5 \mathrm{~W}$ and a peak data throughput of $38 \mathrm{kbps}$ at a range of just over $2,760 \mathrm{~km}$ to a ground station with a $2 \mathrm{~m}$ diameter receiving high-gain antenna with a link margin of $5.9 \mathrm{~dB}$ (Zee and Stibrany, 2002; Pedtke et al., 2004).

This transmitter, unfortunately, is inadequate to the needs of this mission if no carrier relay were involved. For instance, communications with the JUICE spacecraft at a range of 1,000,000 km would require JUICE to possess an HGA with a diameter of $360 \mathrm{~m}$, an unreasonable size for a spacecraft. Alternatively, the power onboard the atmospheric entry probes could be boosted. However, the required output to communicate with the $3 \mathrm{~m}$ diameter HGA of JUICE would be almost $44 \mathrm{~kW}$ - an unreasonable amount of power expenditure for a microsatellite.

Thus, the Carrier spacecraft must be a relay between JUICE and the atmospheric probes. It is not unreasonable for the Carrier to possess an HGA with a diameter of up to $2 \mathrm{~m}$. Furthermore; the addition of a $200 \mathrm{~g}$ amplifier to the MOST transmitters may be capable of bringing its output power up to $5 \mathrm{~W}$. This permits the data rate, which scales linearly with the receive power at the HGA, to be calculated as a function of range, as shown in Figure 4, top panel. Note that the maximum transmit range for MOST was $6,000 \mathrm{~km}$ which suggests that for the $5 \mathrm{~W}$ transmitter described here, the maximum transmit range will be approximately $19,000 \mathrm{~km}$. Similarly, the maximum data rate at zenith of $38 \mathrm{kbps}$ will occur when the spacecraft is less than $8,700 \mathrm{~km}$ distant. Given the favourable transmission power for encounters closer than $8,700 \mathrm{~km}$, it may make sense to add some complexity to the transmitter by permitting variable data rates in order to obtain even more data, at the cost of some additional mass and an increase in necessary pointing accuracy onboard the carrier, however, such a trade study is beyond the scope of this paper. 
Figure 4 (a) Data rate and (b) total data returned as a function of the minimum range between the probes and the carrier spacecraft

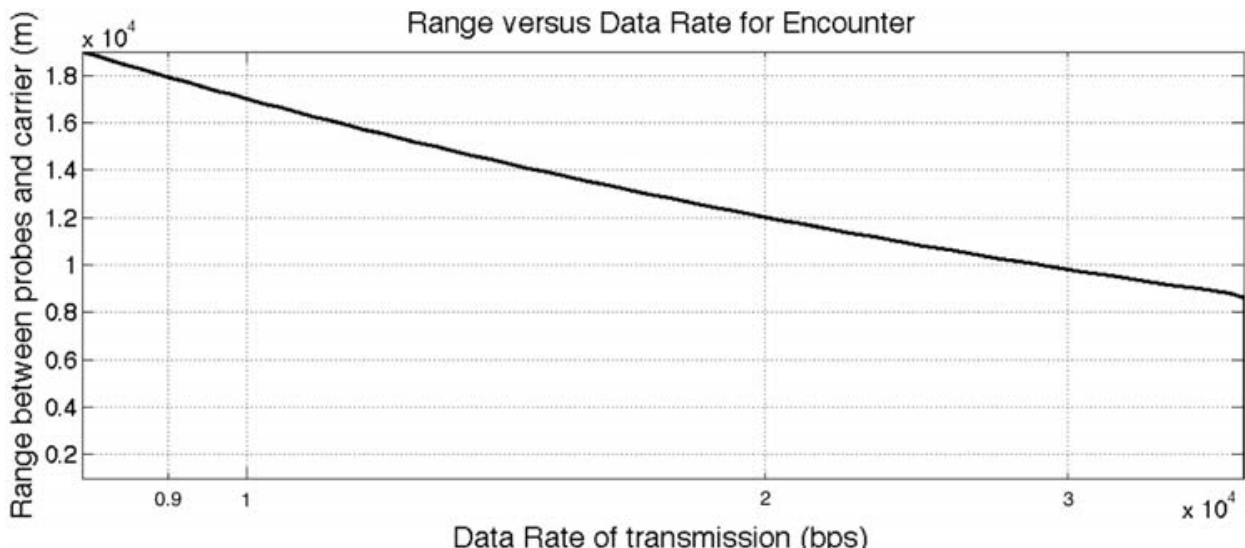

(a)

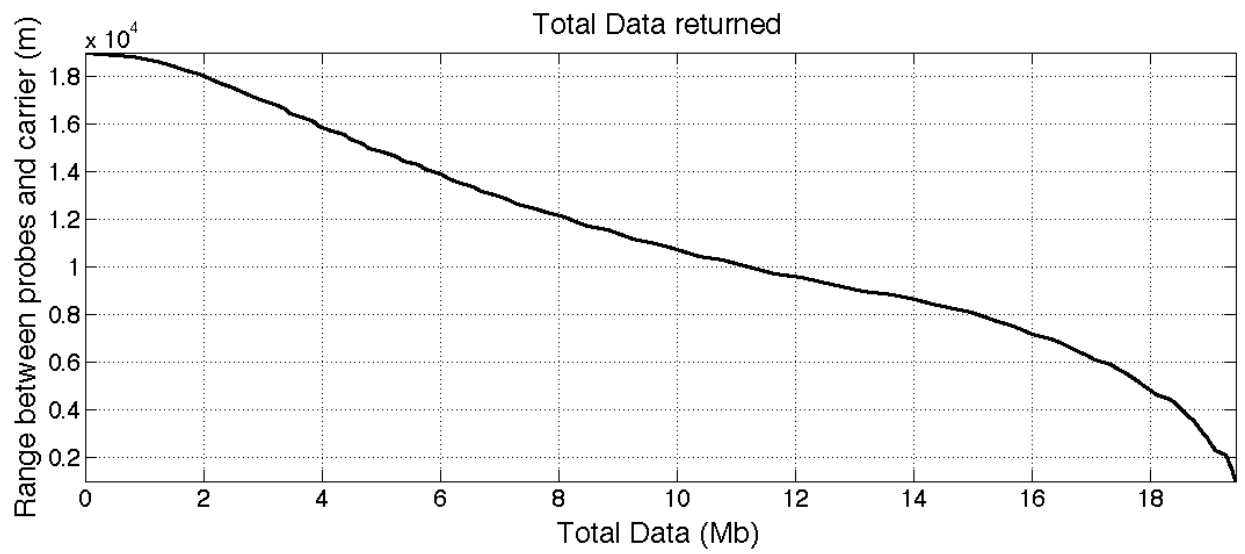

(b)

Notes: Maximum data rates of $38 \mathrm{kbps}$ are achieved at ranges below $8700 \mathrm{~km}$. The maximum data return is $20 \mathrm{Mbits}$, enough for 100 images captured at $256 \times 256$ pixels compressed to $3 \mathrm{bpp}$.

From Figure 4, bottom panel, the sum total of all returned data from the encounter is shown. The greatest returns of 20 Mbit are achieved at a closest approach of 1,000 km, though greater than $12 \mathrm{Mbit}$ will be returned for any approach closer than $10,000 \mathrm{~km}$. Not included in this analysis is the potential advantage of using a smaller transmission angle onboard the probes. This would result in an increase in data rate for approach angles associated with higher carrier orbits near $10,000 \mathrm{~km}$ where the carrier goes out of range at an elevation angle of $35^{\circ}$ - still very high in the sky. This kind of a scheme may ultimately be preferable to an extremely close approach since the closest approaches bias their data return over a short time period around closest approach when the carrier is near local zenith. This means that closest approach must occur late in the fall of the probes. Should the probes fail early, such a scheme risks no data return at all. 
While these data volumes and rates may appear small, they are large compared to other mission designs. For instance, Balint (2005) describes a multiple-probe system in which each individual probe returns only $1.7 \mathrm{Mbit}$ of data over a much longer fall at data rates of 92 to 360 bps. If error checks are included, this rises to 3.4 Mbit total data volume, the best value for a comparison.

\subsubsection{Cruise}

During the cruise phase, the carrier spacecraft communicates with ground stations on the Earth. If the carrier has a transmit power of $20 \mathrm{~W}$, comparable to the Cassini Spacecraft (Spilker, 1997), and possesses a 2 m HGA, the data rate over X-band would be up to 166 kbps, scaling from the performance of Cassini at Saturn (Spilker, 1997). Given that most health-check data is very small, only a few Mbits, communications during cruise through the 70-m receivers of the DSN should not be an issue. In fact, smaller private receivers could be used to command the spacecraft. For instance, the Algonquin Radar Observatory's $46 \mathrm{~m}$ dish could obtain data rates as high as $72 \mathrm{kbps}$. As a result, a much lower powered transmitter could be used onboard the carrier spacecraft. For instance, if a $5 \mathrm{~W}$ transmitter is used, similar to the probes, the data rates fall by a factor of four. The resulting data rates would remain highly favourable.

\subsubsection{Downlink}

Very high data rates may be obtained between the carrier spacecraft and JUICE, each of which is equipped with a large HGA. Such transmissions are likely limited by onboard electronics, as signal theory would predict theoretical data rates in excess of $180 \mathrm{Mbps}$. This would allow all data from a single probe's descent to be transmitted in a fraction of a second.

\section{Mission risks and mitigation strategies}

\subsection{Long lifetime requirement compared to cubesats}

Many small satellite projects are developed with short lifetimes, on the order of months. However, designing to a three year lifespan, for the carrier, or dormancy period, for the probes, introduces complexities that go far beyond operating in LEO. Some of this risk may be mitigated through the removal of potential single-point failures through redundancy. However, in certain cases equipment with enhanced reliability or improved tolerances may need to be substituted. A full analysis of the risk induced by long lifetimes or long dormancies is beyond the scope of this paper, however, there are strategies which may be employed to extend the life of cubesats at little additional cost (Frazier et al., 2013). Furthermore, most cubesats that do not fail during their intitial 'burn-in' phase, typically greatly exceed their lifetime expectations. This suggests that the technology is more robust then would be expected based upon terrestrial lifetime benchmarks. In particular, the cruise phase of the mission will be exceptionally quiescent, especially during long periods in which most equipment is powered off. 


\subsection{Radiation belts at Jupiter}

During entry to and exit from the Jovian system, it will be necessary to pass through the radiation belts. While a polar approach would avoid the worst effects of radiation, this would have a negative effect on the encounter velocity, which is undesirable. An equatorial passage was a part of the Galileo Orbiter Mission and no ill effects were encountered. The Galileo Mission was designed to withstand $150 \mathrm{kRad}$ of radiation dosage over its entire lifetime (Fieseler et al., 2002), a dosage that it substantially exceeded without failure. By comparison, cubesat boards, such as those made by Andrews Space can reliably withstand in excess of $15 \mathrm{kRad}$ (Frazier et al., 2013). This is more than sufficient for the short time the probes and carrier will be present within the Jovian system.

\subsection{Jupiter as a source of radio noise}

The near Jovian environment is a source of intense radio noise (e.g., de Pater et al., 1997), however, this noise is unlikely to pose a problem for the mission architecture described in this paper. Radio emissions by Jupiter are concentrated in three zones; near the north and south poles where magnetic field lines encounter the atmosphere, and in a torus in the equatorial plane of Jupiter, peaking at $\sim 0.5 R_{J}$ from the cloud tops, or approximately $35,000 \mathrm{~km}$ (de Pater et al., 1997). Thus, from the perspective of the individual probes looking up towards the carrier, noise would be considerable if data was needed from the carrier. However, under the mission architecture described here, the probes are intended to passively transmit their data. From the perspective of the carrier looking down towards the individual probes, radio noise should be minor. Thus under a range of conditions from nominal to highly enhanced (e.g., de Pater et al., 1997) noise should not be an issue.

\section{Comparison with previous proposals}

Balint (2005) describes several past mission concept studies for follow-on atmospheric entry probes at Jupiter. In all cases the individual probes selected are large with a minimum mass of $143 \mathrm{~kg}$ for the Jupiter Deep Muliprobes Study carried out by Team X and JPL in 1997 and increasing up to Galileo-like masses in other JPL and ESA CDF (Ritter et al., 2006) Design Studies. An analysis of these previous studies suggests that the factor which drives this large design mass is ultimately the penetration depth required of at least the 100 bar level.

The connection between penetration depth and probe mass is subtle. Once the probes have decelerated to terminal velocity, it was previously determined that fall rates for low aeral density probes, such as the atmospheric entry vehicles described in this paper, would be small. In order for larger, denser spacecraft to spend more time in the low-pressure regime where aerosols are condensing, a parachute is employed which is the first factor which increases the mass and complexity of the entry probe. However, once the 20 bar level is reached, the probe is falling very slowly and it becomes necessary to release the parachute in order for the probe to reach the 100 or 200 bar level in a reasonable amount of time. Balint (2005) calculated this fall time to be 1.88 hours. Even worse, from the perspective of the Carrier Relay, the deepest depth must correspond to 
the highest elevation angle in order to minimise the optical depth at radio wavelengths to the atmospheric probe, thus 1.88 hours describes the time to Zenith only. Taking this hover time into consideration requires the relay spacecraft to have an orbit that takes it out to over 240,000 km from the 1 bar level. Yet, mass concerns aboard the Carrier Relay prevent it from deploying an antenna much bigger than what was specified here in Section 4. As a result, a much larger transmitter $-46 \mathrm{~W}-$ is required onboard the probe to communicate over such large distances. Even with this order of magnitude increase in power, a two orders of magnitude decrease in the data rate must be accepted.

Such capabilities are likely beyond the abilities of small entry probes. While the timing of the entry vehicles and carrier could be adjusted for a deeper penetration at carrier zenith, it is likely that the increased temperature and pressure at depth would be fatal to the kind of probes discussed here. The science conducted by the probes presented here would focus on layers in which condensates would form. The extension from Galileo is in terms of additional techniques applied to this layer and to acquiring additional comparative measurements in time and in geography. Thus the study described in this paper is complimentary to previous work.

\section{Conclusions}

A mission architecture has been described that would allow the deployment of a large number of small entry probes at Jupiter in the 2030 timeframe. Such probes could revolutionise our understanding of the atmosphere of Jupiter at small scales and present a specialised niche in which microsats are the ideal choice for achieving a planetary exploration objective. Such small vehicles will experience less heating during their passage through the atmosphere and therefore this stage of the mission presents less risk than with conventional larger entry probes in the $140 \mathrm{~kg}$ to $300 \mathrm{~kg}$ range.

The amount of data that these entry probes return could be significant - up to 20 Mbits per probe, or enough data to collect 100 images per probe with a resolution of $256 \times 256$ pixels ICER compressed to 3 bits per pixel. Since each probe may carry different instrumentation, a large swarm of such probes could return many different datasets. As well, since each set of probes is organised into groups of 6 probes with a single carrier relaying their signals, each carrier may target a different part of the Jovian atmosphere. As such, it would be possible, in a single launch, to obtain ground truth from a large part of the entire planet. A potential design for the probes themselves will be provided by an article in the next issue of International Journal of Space Science and Engineering (Moores et al., 2014).

\section{References}

Atkinson, D.H., Pollack, J.B. and Seiff, A. (1996) 'Galileo Doppler measurements of the deep zonal winds at Jupiter', Science, Vol. 272, No. 5263, pp.842-843 doi: $10.1126 /$ science.272.5263.842.

Atreya, S.K. and Wong, A-S. (2005) 'Coupled clouds and chemistry of the giant planets - a case for multiprobes', Space Sci Rev., Vol. 16, Nos. 1-2, pp.121-136, doi: 10.1007/s11214-0051951-5. 
Atreya, S.K., Owen, T.C., Bolton, S.J. and Guillot, T. (2006) 'Multiprobe exploration of the giant planets - shallow probes', Proceedings, International Planetary Probe Workshop, IPPW-3, ESA SP-WPP263.

Bairstow, B. and Cataldo, R.L. (2013) 'A low-cost small radioisotope power system centaur flyby smallsat mission concept', Low Cost Planetary Missions Conference, 18-20 June, Pasadena, CA.

Balint, T.S. (2005) 'Overview of mission architecture options for Jupiter deep entry probes', 2005 OPAG Meeting, Boulder CO.

Beebe, R.F., Simon, A.A. and Huber, L.F. (1996) 'Comparison of Galileo probe and earth-based translation rates of Jupiter's equatorial clouds', Science, Vol. 272, No. 5263, p.841

Carroll, K.A., Spencer, H. and Zee, R. (2012) CARAVEL: a solar-sail-based nanosatellite mission to a near-earth asteroid', 2012 Canadian Space Summit, 14-16 November, London, ON.

Castillo-Rogez, J., Klesh, A., Kahn, P., Staehle, R., Nesnas, I. and Pavone, M. (2013) 'Next generation smallsat - dare to explore where no craft has gone before', Low Cost Planetary Missions Conference, 18-20 June, Pasadena, CA.

de Pater, I., Van der Tak, F., Strom, R.G. and Brecht, S.H. (1997) 'The evolution of Jupiter's radiation belts after the impact of comet D/Shoemaker - Levy 9', Icarus, Vol. 129, No. 1, pp.21-47.

Fieseler, P.D., Ardalan, S.M. and Frederickson, A.R. (2002) 'The radiation effects on Galileo spacecraft systems at Jupiter', IEEE Transactions on Nuclear Science, Vol. 49, No. 6, pp.2739-2758, doi: 10.1109/TNS.2002.805386.

Fischer, H.M., Pehlke, E., Wibberenz, G., Lanzerotti, L.J. and Mihalov, J.D. (1996) 'High energy charged particles in the innermost Jovian magnetosphere', Science, Vol. 272, No. 5263, pp.856-858.

Fortney, J.J. and Hubbard, W.B. (2003) 'Phase separation in giant planets: inhomogeneous evolution of Saturn', Icarus, Vol. 164, No. 1, pp.228-243, Doi: 10.1016/S0019-1035(03)00130-1.

Frazier, W., Rohrschneider, R. and Verzuh, M. (2013) 'Cubesat strategies for long-life missions', Low Cost Planetary Missions Conference, 18-20 June, Pasadena, CA.

Garrick- Bethell, I. and 12 co-authors (2013) 'Lunar magnetic field measurements with a cubesat impactor', Low Cost Planetary Missions Conference, 18-20 June, Pasadena, CA.

Halatek, L. (2013) 'Cubesats and Europa: focused science with disposable spacecraft', Low Cost Planetary Missions Conference, 18-20 June, Pasadena, CA.

Hunten, D.M., Colin, L. and Hansen, J.E. (1986) 'Atmospheric science on the Galileo mission', Space Science Reviews, Vol. 44, Nos. 3-4, pp.191-240.

Komarek, T., Bailey, Z., Schone, H., Jedrey, T. and Chandler, A. (2013) 'Novel ideas for exploring mars with CubeSats: challenges and possibilities', Low Cost Planetary Missions Conference, 18-20 June, Pasadena, CA.

Lang, J.J., Baker, J.D., McElrath, T.P., Moreno, T. and Snyder, J.S. (2013) 'Enabling low cost planetary missions through rideshare opportunities', Low Cost Planetary Missions Conference, 18-20 June, Pasadena, CA.

Lanzerotti, L.J., Rinnert, K., Dehmel, G., Gliem, F.O., Krider, E.P., Uman, M.A. and Bach, J. (1996) 'Radio frequency signals in Jupiter's atmosphere', Science, Vol. 272, No. 5263, pp.858-860.

Lloyd, J. (2013) 'Far above: interplanetary dust structures with a small satellite in inclined heliocentric orbit', Low Cost Planetary Missions Conference, 18-20 June, Pasadena, CA.

Matousek, S. (2007) 'The Juno new frontiers mission', Acta Astronautica, Vol. 61, No. 10, pp.932-939.

Mitri, G. (2002) 'Preliminary design for a Europa mission', Proceedings of the 33rd Lunar and Planetary Science Conference, Clear Lake, TX, Abstract no. 1530. 
Moores, J.E., Carroll, K.A., DeSouza, I., Sathiyanathan, K., Stoute, B., Shan, J., Lee, R.S. and Quine, B. (2014) 'The small reconnaissance of atmospheres mission platform concept, part 2: design of carrier spacecraft and atmospheric entry probes', Int. J. Space Science and Engineering, Vol. 2, No. 4, pp.345-364.

National Research Council Visions and Voyages for Planetary Science in the Decade 2013-2022 (2011) Ed S. Squyres, ISBN 978-0-309-22464-2.

Niemann, H.B. and 12 co-authors (1996) 'The Galileo probe mass spectrometer: composition of Jupiter's atmosphere', Science, Vol. 272, No. 5263, pp.846-849.

Orton, G. and 40 co-authors (1996) 'Earth-based observations of the Galileo probe entry site', Science, Vol. 272, No. 5263, pp.839-840.

Pedtke, D., Lofquist, M. and Kohlhepp, K. (2004) 'The modular S-Band radio suite', AIAA Small Satellite Conference, Abstract 26.

Poncy, J., Couzin, P. and Billot, C. (2013) 'Using smallsats and cubesats as ancillaries: a low-cost strategy maximizing the science return of fly-by missions', Low Cost Planetary Missions Conference, 18-20 June, Pasadena, CA.

Ragent, B., Colburn, D.S., Avrin, P. and Rages, K.A. (1996) 'Results of the Galileo probe nephelometer experiment', Science, Vol. 272, No. 5263, pp.854-856.

Ragent, B., Colburn, D.S., Rages, K.A., Knight, T.C.D., Arvin, P., Orton, G.S., Yanamandra-Fischer, P.A. and Grams, G.W. (1998) 'The clouds of Jupiter: results of the Galileo Jupiter mission probe nephelometer experiment', J. Geopys. Res., Vol. 103, No. E10, pp.22891-22910, doi: 10.1029/98JE00353.

Riedel, J.E., Marrese-Reading, C. and Lee, Y.H. (2013) 'A low-cost NEO micro Hunter-Seeker mission concept', Low Cost Planetary Missions Conference, 18-20 June, Pasadena, CA.

Ritter, H., Mazoué, F., Santovincenzo, A. and Atzei, A. (2006) 'Jupiter entry probe feasibility study from the ESTEC CDF team heat flux evaluation and TPS definition', 5th European Workshop on Thermal Protection Systems and Hot Structures, Noordwijk, Netherlands.

Seiff, A. and 10 co-authors (1996) 'Structure of the atmosphere of Jupiter: Galileo probe measurements', Science, Vol. 272, No. 5263, pp.844-845.

Spencer, H. and Zee, R. (2012) 'MOMENT: a Canadian Nanosatellite Mars Orbiter for magnetic mapping', 2012 Canadian Space Summit, 14-16 November, London, ON.

Spilker, L.J. (1997) Passage to a Ringed World: The Cassini-Huygens Mission to Saturn and Titan, NASA document SP-533, Washington DC.

Sromovsky, L.A., Best, F.A., Collard, A.D., Fry, P.M., Revercomb, H.E., Freeman, R.S., Orton, G.S., Hayden, J.L., Tomasko, M.G. and Lemmon, M.T. (1996) 'Solar and thermal radiation in Jupiter's atmosphere: initial results of the Galileo probe net flux radiometer', Science, Vol. 272, No. 5263, pp.851-854.

Von Zahn, U. and Hunten, D.M. (1996) 'The helium mass fraction in Jupiter's atmosphere', Science, Vol. 272, No. 5263, pp.849-851.

Worden, P. (2012) 'Small satellites for science and other uses: promises and challenges', 2012 Canadian Space Summit, 14-16 November, London, ON.

Young, R.E., Smith, M.A. and Sobeck, C.K. (1996) 'Galileo probe: in situ observations of Jupiter's atmosphere', Science, Vol. 272, No. 5263, pp.837-838.

Zee, R.E. and Stibrany, P. (2002) 'Canada's first microsatellite - an enabling low-cost technology for future space science and technology missions', Canadian Aeronautics and Space Journal, Vol. 48, No. 1, pp.1-11, 10.5589/q02-008. 